\title{
Multicentric Reticulohistiocytosis (MRH): First Case Report with Tenosynovial Fluid Analysis and Tenosynovial Histology Demonstrating the Classic Histologic Findlings
}

\author{
Megha Sawhney ${ }^{1}$, Peter Levitin ${ }^{2}$ \\ ${ }^{1}$ National Institutes of Arthritis, Musculoskeletal and Skin Disease, National Institutes of Health, Bethesda, USA \\ ${ }^{2}$ Eagle Physicians \& Associates, Greensboro, NC/Clinical Professor of Medicine, Department of Medicine, \\ University of North Carolina School of Medicine, Chapel Hill, NC, USA \\ Email: megha.sawhney@nih.gov, plevi21@aol.com
}

Received 16 February 2016; accepted 9 May 2016; published 12 May 2016

Copyright (C 2016 by authors and Scientific Research Publishing Inc.

This work is licensed under the Creative Commons Attribution International License (CC BY).

http://creativecommons.org/licenses/by/4.0/

\section{(c) (i) Open Access}

\begin{abstract}
Multicent ricreticulohistiocytosis (MRH) is a rare systemic disease of unclear etiology characterized by destructive, deforming arthritis, nodules in the skin, mucous membrane and internal organs and can be associated with malignancy. The tenosynovial fluid and tenosynovium histologic findings have not been reported in any case reports of MRH in the literature. To our knowledge, this is the first case report of tenosynovial fluid and tenosynovium demonstrating the classic histologic findings of histiocytes with a foamy eosinophilic cytoplasm. This case also demonstrates a non-deforming arthritis.
\end{abstract}

Keywords

Tenosynovitis, Tenosynovial Fluid Analysis

\section{Introduction}

Multicent ricreticulohistiocytosis (MRH) is a rare systemic disease of unclear etiology characterized by inflammatory arthritis, nodules in the skin, mucous membrane and internal organs and can be associated with malignancy [1] [2]. Arthritis can be destructive and deforming progressing to arthritis mutilans in majority of the cas- 
es but generally remit spontaneously in 10 years. Tenosynovial involvement may also occur [3]. The tenosynovial fluid and tenosynovium histologic findings have not been reported in any case reports of MRH in the literature.

\section{Case Report}

A 27 year old woman with no significant past medical history presented with 3 month history of bilateral finger, wrist and shoulder pain with intermittent numbness and tingling of both hands. A month prior to her office visit, she noticed a pale 'knot' over the base of her left thumb. Initial evaluation revealed tenosynovitis of the left wrist, decreased flexion and extension in second and third PIP joints bilaterally and a nodule over the left extensor pollicis brevis. Laboratory studies revealed a WBC of $5400 / \mu \mathrm{L}$ with $60 \%$ neutrophils (normal $34 \%$ $71 \%)$, 28\% lymphocytes (19.3\% - 51.7\%), 4\% eosinophils (0.7\% - 5.8\%), 7\% monocytes ( $4.7 \%$ - 12.5\%), hemoglobin of $15 \mathrm{~g} / \mathrm{dL}(11.2$ - $15.7 \mathrm{~g} / \mathrm{dl})$, hematocrit of $45 \%(34.1 \%-44.9 \%)$, platelets $212 \mathrm{k} / \mu \mathrm{L}(173-369$ $\mathrm{K} / \mu \mathrm{L})$, erythrocyte sedimentation rate of $6 \mathrm{~mm} / \mathrm{hr}(0-42 \mathrm{~mm} / \mathrm{hr})$ and C-reactive protein of $0.9 \mathrm{mg} / \mathrm{dl}(0-1.0$ $\mathrm{mg} / \mathrm{dl})$. Comprehensive metabolic panel, lipids, thyroid, serum and urine immunoelectrophoresis were within normal limits. ANA, anti-ds DNA and RF were negative. X-ray of hands and wrist were normal.

Three weeks after the initial visit, the patient developed new nodules on the volar surface of left second and fifth fingers. The tenosynovium of the left wrist was aspirated and revealed about $0.5 \mathrm{cc}$ of clear yellow colored fluid. The tenosynovial fluid analysis was negative for monosodium urate and calcium pyrophosphate crystals, gram stain showed no organism and cultures were negative. Fluid was insufficient for cell count but smear showed many white cells predominantly mononuclear.

Over the next six months, the patient developed further small skin nodules involving distal interphalangeal (DIP) and proximal interphalangeal (PIP) joints bilaterally, periungual areas of the nails, forehead, olecranon, and mucosal nodules under the tongue. Because of the appearance of the nodules, joint symptoms and no definite diagnosis, a diagnostic biopsy of the forehead and right third finger nodule was done that was reported as xanthoma. With the appearance and distribution of nodules involving the fingers, a diagnosis of MRH was considered. The original slides were reviewed and additional stains were applied. An oil red stain for fat was negative ruling out xanthoma. The cytoplasm stained positive with PAS stain and resistant to diastase, which was consistent with diagnosis of MRH. The patient was treated with NSAIDs which helped with arthralgias but the bilateral tenosynovitis and bilateral numbness of both hands continued. EMG results of right and left wrist were consistent with bilateral carpal tunnel syndrome. Each carpal tunnel was injected corticosteroid with partial relief of symptoms. Left dorsal tenosynovitis was persistent and aspiration was done that revealed histiocytes. Because of persistent tenosynovitis and carpal tunnel symptoms, the patient underwent bilateral tenosynovectomy with carpal tunnel release. The tenosynovium was sent to pathology and the results showed classic histologic findings of proliferation of synovial villi with foamy eosinophilic cytoplasm and multinucleated giant cells consistent with MRH.

One year later the patient developed right axillary lymphadenopathy and a biopsy showing stage Ia Histiocytoma treated successful with radiotherapy.

When the patient followed up in the clinic almost 20 years after initial diagnosis-there was no synovitis or tenosynovitis and the only finding was triggering from left second through fifth fingers.

\section{Discussion}

MRH is an idiopathic disease of unclear etiology characterized by destructive polyarthritis and papulonodular mucocutaneous lesions [1]-[3]. It was first reported in 1937 by Weber and Freudenthal [4] and fewer than 300 cases have been reported in the literature thus far [5]. It predominantly affects women in fifth and sixth decades of life. MRH presents insidiously with joint symptoms preceding cutaneous disease in two-thirds of the patients and in the remainder of patients cutaneous findings appear as the initial symptom or occur simultaneously with the joint symptoms [6] [7]. The disease usually has rapid progression of arthritis which is considered as one of the hallmarks of the disease and progresses to arthritis mutilans in $45 \%$ of the cases [8].

The tendon sheaths on the flexor and extensor surfaces of the wrists may be involved, and Duputyren's contracture or carpal tunnel syndrome has been described [1]. Histopathology findings of skin nodules and synovium are similar and consist of large histiocytes and multinucleate giant cells with eosinophilic ground glass cytoplasm, that stain positive with periodic-acid Schiff (PAS) and are diastase resistant. It has been associated 
with malignancy in 25\% of cases [1] [9]. A firm diagnosis of MRH was made before neoplasm was discovered in $73 \%$ of subjects [10].

While synovial fluid and synovial biopsy findings showing histiocytes have been reported in the literature [11] [12], there have not been any case reports discussing tenosynovial fluid and biopsy findings. Our patient presented with wrist tenosysnovitis and underwent tenosynovial fluid analysis and tenosynovial biopsy demonstrated the classic multinucleated giant cells with foamy eosinophilic cytoplasm. The patient also developed malignancy, which can be seen with MRH.

Our patient had very good response to NSAIDS which is still considered as first line treatment in addition to steroids but now biological agents like TNF inhibitors have been also shown to be successful treatment options based on the observed levels of proinflammatory cytokinesin MRH tissues [13]. In addition promising results have also been achieved using cytotoxic drugs like cyclophosphamide in various case reports.

The unusual feature of our case was its benign non-destructive arthritis unlike the progressive destructive course of arthritis usually seen in multicent ricreticulohistiocytosis.

\section{Conclusion}

To our knowledge, this is the first case report of tenosynovial fluid and tenosynovium histopathology demonstrating histiocytes. In conclusion, our case is unique with tenosynovial fluid and tenosynovium histopathology showing histiocytes.

\section{References}

[1] Wieisman, M.H. (1979) Arthritis Associated with Hematologic Disorders, Storage Diseases, Disorders of Lipid Metabolism, and Dysproteinemias. Arthritis and Allied Condition. Textbook of Rheumatology, McCarty, D.J., Ed., 932934.

[2] Barrow, M.V. and Holubar, K. (1969) Multicent Ricreticulohistiocytosis: A Review of 33 Patients. Medicine (Baltimore), 48, 287-305. http://dx.doi.org/10.1097/00005792-196907000-00002

[3] Melton $3^{\text {rd }}$, J.W. and Irby, R. (1972) Multicentric Reticulohistiocytosis. Arthritis \& Rheumatology, 15, $221-226$. http://dx.doi.org/10.1002/art.1780150215

[4] Weber Parkes, F. and Freudenthal, W. (1937) Nodular Non-Diabetic Cutaneous Xanthomatosis with Hypercholesterolemia and Atypical Histological Features. Proc R Soc Med., 30, 522-526.

[5] Han, L., Huang, Q., Liao, K.H., Chen, L.J., Kong, W.Y., Fu, W.W. and Xu, J.H. (2012) Multicentric Reticulohistiocytosis Associated with Liver Carcinoma: Report of a Case. Case Reports in Dermatology, 4, 163-169. http://dx.doi.org/10.1159/000341563

[6] Klippel, J.H., Crofford, L. and Gordon, D.A. (2008) Storage and Deposition Diseases. Primer on Rheumatic Diseases. Text Book of Rheumatology, 13th Edition, Springer, 530-531.

[7] Toporcer, M.B., Kantor, G.R. and Benedetto, A.V. (1991) Multiple Cutaneous Reticulohistiocytomas (Reticulohistiocytic Granulomas). Journal of the American Academy of Dermatology, 25, 948-951. http://dx.doi.org/10.1016/0190-9622(91)70291-9

[8] Gorman, J.D. (2000) Case Report with Immunohistochemical Analysis and Literature Review. Arhritis and Rheumatism, 43, 930-938.

[9] Sroa, N., Zirwas, M.J. and Bechtel, M. (2010) A Case Report and Review of Literature. Cutis, 85, 153-155.

[10] Nunnink, J.C., Krusinski, P.A. and Yates, J.W. (1985) Multicent Ricreticulohistiocytosis and Cancer: A Case Report and Review of the Literature. Medical and Pediatric Oncology, 13, 273-279. http://dx.doi.org/10.1002/mpo.2950130506

[11] Krey, P.R., Comerford, F.R. and Cohen, A.S. (1974) Multicentric Reticulohistiocytosis: Fine Structural Analysis of the Synovium and Synovial Fluid Cells. Arthritis \& Rheumatology, 17, 615-633. http://dx.doi.org/10.1002/art.1780170518

[12] Freemont, A.J., Jones, C.J. and Denton, J. (1983) The Synovium and Synovial Fluid in Multicent Ricreticulohistiocytosis-A Light Microscopic, Electron Microscopic and Cytochemical Analysis of One Case. Journal of Clinical Pathology, 36, 860-866. http://dx.doi.org/10.1136/jcp.36.8.860

[13] Bennássar, A., Mas, A., Guilabert, A., Juliá, M., Mascaró-Galy, J.M. and Herrero, C. (2011) Multicent Ricreticulohistiocytosis with Elevated Cytokine Serum Levels. J Dermatol, 38, 905-910. 